\title{
Rattus norvegicus BN/SHR liver and heart left ventricle ribosomal RNA depleted directional RNA sequencing
}

\author{
Emanuel Wyler ${ }^{1 *} \mathbb{D}$, Sebastiaan van Heesch², Eleonora Adami ${ }^{2}$, Norbert Hubner $^{2}$ and Markus Landthaler ${ }^{1}$
}

\begin{abstract}
Objective: The spontaneously hypertensive rat strain is a frequently used disease model. In a previous study, we measured translational efficiency from this strain and BN- $L x$ animals. Here, we describe long RNA sequencing reads from ribosomal RNA depleted samples from the same animals. This data can be used to investigate splicing-related events.
\end{abstract}

Results: RNA was extracted from rat liver and heart left ventricle from BN-Lx and SHR/Ola rats in biological replicates. Ribosomal RNA was removed and the samples subjected to directional high-throughput RNA-sequencing. Read and alignment statistics indicate high quality of the data. The raw sequencing reads are freely available on the NCBI short read archive and can be used for further research on tissue and strain differences, or analysed together with other published high-throughput data from the same animals.

Keywords: Rat, RNA, Sequencing, BN-Lx, SHR, Liver, Heart

\section{Introduction}

The inbred rat strain SHR/Ola (spontaneously hypertensive rat) is frequently used to study cardiovascular and metabolic diseases [1]. In a previous study, we investigated translational regulation in this strain compared to the BN-Lx reference strain [2], in which small mRNA fragments were sequenced alongside the ribosome-protected fragments. However, since these sequencing reads are on average only 40 nucleotides long, we proceeded to generate also long RNA sequencing reads from the same animals. This enables investigation of features that are more depending on mapping length, such as splicing, circular RNA detection, or variant calling.

\section{Main text}

Total RNA was isolated from frozen, pulverized liver and heart left ventricle. Here, we describe the ribosomal RNA-depleted RNA sequencing obtained from these

\footnotetext{
*Correspondence: emanuel.wyler@mdc-berlin.de

1 Berlin Institute for Medical Systems Biology, Max Delbrück Center

for Molecular Medicine, 13125 Berlin, Germany

Full list of author information is available at the end of the article
}

samples. An overview with SRA and BioSample identifiers is given in Table 1. The animal identifiers here (BN03 etc.) correspond to the previously used identifiers [2], which can be found in the BioProject https://www.ncbi. nlm.nih.gov/bioproject/PRJEB7498.

The Illumina Ribo-Zero kit (Illumina Cat.-No. MRZH11124) was applied to remove ribosomal RNA and the sequencing libraries were prepared using the Illumina TruSeq Stranded mRNA Library Prep Kit (Illumina

Table 1 Overview of sequenced samples

\begin{tabular}{lll}
\hline Description & BioSample ID & SRA ID \\
\hline BN-03 liver & SAMN04543787 & SRS1884865 \\
BN-03 heart left ventricle & SAMN04543786 & SRS1884868 \\
BN-04 liver & SAMN04543789 & SRS1884867 \\
BN-04 heart left ventricle & SAMN04543788 & SRS1884866 \\
SHR-02 liver & SAMN04543791 & SRS1884869 \\
SHR-02 heart left ventricle & SAMN04543790 & SRS1884870 \\
SHR-03 liver & SAMN04543793 & SRS1884871 \\
SHR-03 heart left ventricle & SAMN04543792 & SRS1884872 \\
\hline
\end{tabular}


Cat.-No. RS-122-2101). The raw sequencing reads can be downloaded from the NCBI SRA (short read archive) under the accession number SRP095829 (https://www. ncbi.nlm.nih.gov/sra/?term=SRP095829), or accessed through the NCBI BioProject PRJNA314751 (https:// www.ncbi.nlm.nih.gov/bioproject/PRJNA314751). Per sample, 30-40 million reads were obtained. The adapter sequence GATCGGAAGAGCACACGT was trimmed using Flexbar [3] and trimmed reads with 18 nucleotides or longer were aligned to version rn6 of the rat genome in conjunction with the Ensembl annotation version 81 using tophat2 [4]. The numbers of raw, trimmed, aligned, and unique aligned reads are shown in Fig. 1. The absolute and relative numbers of aligned and unique aligned reads are shown alongside the number of reads mapping to the rat $45 \mathrm{~S}$ ribosomal RNA in Table 2.

To quantify the de novo detection of splice sites, we applied the FindCirc pipeline [5], which identifies backsplicing events indicative of circular RNAs. Comparing the long RNA reads presented here with the smaller RNA fragments that were sequenced alongside the ribosome protected fragments [2], we found the following improvements in the average numbers of detected back-splice junctions per sample: for liver samples, 273 vs. 38, and for heart left ventricle, 679 vs. 61 .

In summary, we provide here a high quality RNA sequencing dataset for two rat strains, with two different tissues from two different animals for each strain. This data can be used to analyze differences between tissues and strains, e.g. variant calling, de novo transcriptome assembly, differential expression and splicing, or circRNA detection. Since the samples originate from the same animals as the ones in the study about translational differences between a healthy rat (BN$L x)$ and a model for hypertensive cardiomyopathy and metabolic syndrome (SHR) [2], the ribosome profiling data from that study can readily be integrated into the analysis.

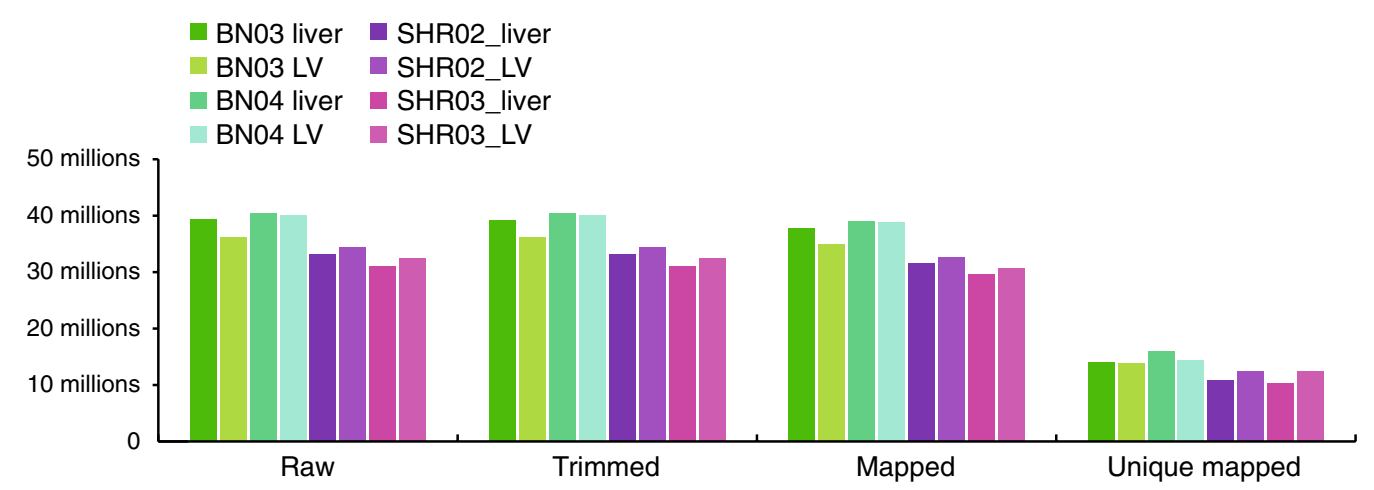

Fig. 1 Number of reads of the eight RNA sequencing samples described here. Shown are, from left to right: raw read numbers; reads after adapter trimming and removal of reads shorter than 18 nucleotides; reads mapping to the rat genome/transcriptome; unique reads mapping to the rat genome/transcriptome

Table 2 Alignment statistics

\begin{tabular}{llllr}
\hline Description & $\begin{array}{l}\text { Aligned reads (absolute } \\
\text { numbers and percent } \\
\text { of trimmed reads) }\end{array}$ & $\begin{array}{l}\text { Unique alignments } \\
\text { (absolute numbers } \\
\text { and percent of aligned } \\
\text { reads) }\end{array}$ & $\begin{array}{l}\text { Non-duplicated aligned } \\
\text { reads (absolute numbers } \\
\text { and percent of aligned } \\
\text { reads) }\end{array}$ & $\begin{array}{l}\text { Reads aligning to ribosomal RNA } \\
\text { (absolute numbers and percent } \\
\text { of trimmed reads) }\end{array}$ \\
\hline BN-03 liver & $37,870,875(96.4 \%)$ & $32,882,723(86.8 \%)$ & $14,091,152(37.2 \%)$ & $805,959(2.1 \%)$ \\
BN-03 heart left ventricle & $34,925,794(96.6 \%)$ & $30,737,415(88.0 \%)$ & $13,866,921(39.7 \%)$ & $211,474(0.6 \%)$ \\
BN-04 liver & $39,082,130(96.8 \%)$ & $34,683,358(88.7 \%)$ & $15,931,304(40.8 \%)$ & $181,485(0.5 \%)$ \\
BN-04 heart left ventricle & $38,793,389(96.6 \%)$ & $33,970,899(87.6 \%)$ & $14,413,293(37.2 \%)$ & $53,931(0.1 \%)$ \\
SHR-02 liver & $31,681,912(95.4 \%)$ & $27,079,893(85.5 \%)$ & $10,814,241(34.1 \%)$ & $151,962(0.5 \%)$ \\
SHR-02 heart left ventricle & $32,713,041(95.0 \%)$ & $28,471,299(87.0 \%)$ & $12,435,350(38.0 \%)$ & $284,145(0.8 \%)$ \\
SHR-03 liver & $29,610,437(95.5 \%)$ & $25,567,357(86.3 \%)$ & $10,308,328(34.8 \%)$ & $201,844(0.7 \%)$ \\
SHR-03 heart left ventricle & $30,803,403(94.94 \%)$ & $27,026,756(87.7 \%)$ & $12,470,719(40.4 \%)$ & $65,253(0.2 \%)$ \\
IIlumina human body & $45,896,887(94.5 \%)$ & $42,741,402(93.1 \%)$ & $10,370,858(22.6 \%)$ & $548,720(1.1 \%)$ \\
$\quad \begin{array}{l}\text { map 2.0 liver (SRA acces- } \\
\text { sion number ERR030895) }\end{array}$ & & & & \\
\hline
\end{tabular}




\section{Limitations}

- The data described here is limited to two organs, heart left ventricle and liver.

- The sequencing depth does not allow for reliable quantification and characterization of very low abundant transcripts.

- Genomic DNA was not sequenced, and has to be used from other animals of the same strains.

\section{Abbreviations}

$B N-L x$ : brown Norway rat strain carrying the polydactyly-luxate syndrome $L x$ allele; SHR: spontaneous hypertension rat; SRA: short read archive.

\section{Authors' contributions}

$\mathrm{SVH}$ and EA collected the samples and isolated RNA. EW prepared sequencing libraries, analysed the data and wrote the manuscript. NH and ML supervised the work. All authors read and approved the final manuscript.

\section{Author details}

${ }^{1}$ Berlin Institute for Medical Systems Biology, Max Delbrück Center for Molecular Medicine, 13125 Berlin, Germany. ${ }^{2}$ Cardiovascular and Metabolic Sciences, Max Delbrück Center for Molecular Medicine, 13125 Berlin, Germany.

\section{Acknowledgements}

We thank Ouidad Benlasfer and Madlen Sohn for excellent technical assistance.

\section{Competing interests}

The authors declare that they have no competing interests.

\section{Availability of data and materials}

The dataset described here is available in the NCBI/SRA repository, https:// www.ncbi.nlm.nih.gov/sra/?term=SRP095829.

\section{Consent for publication}

Not applicable.

\section{Ethics approval and consent to participate}

Animals were housed, bred and fed ad libitum in an air-conditioned animal facility at the Czech Academy of Sciences, Prague, Czech Republic. All experimental procedures were carried out in accordance with the European Union National Guidelines and the Animal Protection Law of the Czech Republic (311/1997) and were approved by the Ethics Committee of the Institute of Physiology, Czech Academy of Sciences, Prague.

\section{Funding}

Not applicable.

\section{Publisher's Note}

Springer Nature remains neutral with regard to jurisdictional claims in published maps and institutional affiliations.

Received: 11 January 2017 Accepted: 29 July 2017

Published online: 11 August 2017

\section{References}

1. Okamoto K, Aoki K. Development of a strain of spontaneously hypertensive rats. Jpn Circ J. 1963;27:282-93.

2. Schafer S, Adami E, Heinig M, Rodrigues KE, Kreuchwig F, Silhavy J, van Heesch S, Simaite D, Rajewsky N, Cuppen E, et al. Translational regulation shapes the molecular landscape of complex disease phenotypes. Nat Commun. 2015;6:7200

3. Dodt M, Roehr JT, Ahmed R, Dieterich C. FLEXBAR-flexible barcode and adapter processing for next-generation sequencing platforms. Biology. 2012;1(3):895-905.

4. Kim D, Pertea G, Trapnell C, Pimentel H, Kelley R, Salzberg SL. TopHat2: accurate alignment of transcriptomes in the presence of insertions, deletions and gene fusions. Genome Biol. 2013;14(4):R36.

5. Memczak S, Jens M, Elefsinioti A, Torti F, Krueger J, Rybak A, Maier L, Mackowiak SD, Gregersen LH, Munschauer M, et al. Circular RNAs are a large class of animal RNAs with regulatory potency. Nature. 2013:495(7441):333-8.

\section{Submit your next manuscript to BioMed Central} and we will help you at every step:

- We accept pre-submission inquiries

- Our selector tool helps you to find the most relevant journal

- We provide round the clock customer support

- Convenient online submission

- Thorough peer review

- Inclusion in PubMed and all major indexing services

- Maximum visibility for your research

Submit your manuscript at www.biomedcentral.com/submit 